\title{
Time course of changes in heart rate and blood pressure variability in streptozotocin-induced diabetic rats treated with insulin
}

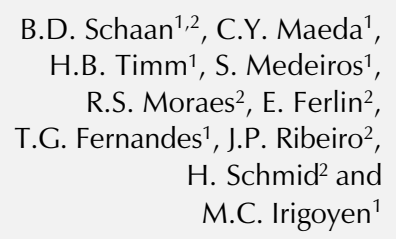

Correspondence

M.C. Irigoyen

Departamento de Fisiologia

Instituto de Biociências, UFRGS

Rua Sarmento Leite, 500

90050-170 Porto Alegre, RS

Brasil

Presented at the XI Annual Meeting of the Federação de Sociedades de Biologia Experimental, Caxambu, MG, Brasil, August 21-24, 1996.

Research supported by CNPq, FIPE-HCPA, FAPERGS, and CAPES.

Received April 19, 1996 Accepted July 15, 1997

\author{
'Laboratório de Fisiologia Cardiovascular, Instituto de Biociências, \\ Universidade Federal do Rio Grande do Sul, Porto Alegre, RS, Brasil \\ ${ }^{2}$ Serviços de Endocrinologia, Cardiologia e Engenharia Biomédica, \\ Hospital de Clínicas de Porto Alegre, Universidade Federal do Rio Grande do Sul, \\ Porto Alegre, RS, Brasil
}

\section{Abstract}

Autonomic neuropathy is a frequent complication of diabetes associated with higher morbidity and mortality in symptomatic patients, possibly because it affects autonomic regulation of the sinus node, reducing heart rate (HR) variability which predisposes to fatal arrhythmias. We evaluated the time course of arterial pressure and HR and indirectly of autonomic function (by evaluation of mean arterial pressure (MAP) variability) in rats (164.5 $\pm 1.7 \mathrm{~g}) 7,14,30$ and 120 days after streptozotocin (STZ) injection, treated with insulin, using measurements of arterial pressure, HR and MAP variability. HR variability was evaluated by the standard deviation of $R R$ intervals (SDNN) and root mean square of successive difference of RR intervals (RMSSD). MAP variability was evaluated by the standard deviation of the mean of MAP and by 4 indices $\left(\mathrm{P}_{1}, \mathrm{P}_{2}, \mathrm{P}_{3}\right.$ and $\left.\mathrm{MN}\right)$ derived from the three-dimensional return map constructed by plotting $\mathrm{MAP}_{\mathrm{n}}$ $\mathrm{x}\left[\left(\mathrm{MAP}_{\mathrm{n}+1}\right)-\left(\mathrm{MAP}_{\mathrm{n}}\right)\right] \mathrm{x}$ density. The indices represent the maximum concentration of points $\left(\mathrm{P}_{1}\right)$, the longitudinal axis $\left(\mathrm{P}_{2}\right)$, and the transversal axis $\left(\mathrm{P}_{3}\right)$ and $\mathrm{MN}$ represents $\mathrm{P}_{1} \times \mathrm{P}_{2} \times \mathrm{P}_{3} \times 10^{-3}$. STZ induced increased urinary glucose in diabetic (D) rats compared to controls (C). Seven days after STZ, diabetes reduced resting HR from $380.6 \pm$ 12.9 to $319.2 \pm 19.8 \mathrm{bpm}$, increased HR variability, as demonstrated by increased SDNN, from $11.77 \pm 1.67$ to $19.87 \pm 2.60 \mathrm{~ms}$, did not change MAP, and reduced $\mathrm{P}_{1}$ from $61.0 \pm 5.3$ to $51.5 \pm 1.8$ arbitrary units (AU), $\mathrm{P}_{2}$ from $41.3 \pm 0.3$ to $29.0 \pm 1.8 \mathrm{AU}$, and $\mathrm{MN}$ from 171.1 \pm 30.2 to $77.2 \pm 9.6$ AU of MAP. These indices, as well as HR and MAP, were similar for D and C animals 14, 30 and 120 days after STZ. Seven-day rats showed a negative correlation of urinary glucose with resting $\mathrm{HR}(\mathrm{r}=-0.76, \mathrm{P}=0.03)$ as well as with the $\mathrm{MN}$ index $(\mathrm{r}=-0.83$, $\mathrm{P}=0.01)$. We conclude that rats with short-term diabetes mellitus induced by STZ presented modified autonomic control of HR and MAP which was reversible. The metabolic control may influence these results, suggesting that insulin treatment and a better metabolic control in this model may modify arterial pressure, HR and MAP variability.
Key words

- Diabetes mellitus

- Streptozotocin

- Insulin

- Autonomic nervous system 
Autonomic neuropathy is a frequent complication of diabetes mellitus associated with higher morbidity and mortality in symptomatic patients (1) which affects the autonomic modulation of the sinus node, reducing heart rate (HR) variability (2). In addition, these patients have a higher systolic blood pressure during 24-h monitoring (3). In the rat model, Monckton and Pehowich (4) described degenerative changes in autonomic neurons occurring from 3 days to 6 weeks after the injection of streptozotocin (STZ). Other studies have shown degenerative changes 5 to 15 months after the use of STZ (5-7). In our laboratory, we recently observed that STZ-induced diabetic rats with no insulin treatment presented lower mean arterial pressure (MAP) and HR than controls, as well as reduced intrinsic HR, vagal and sympathetic tone and sympathetic effect, suggesting early autonomic dysfunction due to diabetes (8). Little information is available on the time course of changes in autonomic control in STZ-induced diabetic rats treated with insulin.

The autonomic nervous system modulates beat-to-beat fluctuations in heart rate and blood pressure, playing an important role in cardiovascular homeostasis. In the healthy state, heart rate variability is high at rest and reduced during adrenergic stimulation, while blood pressure variability is reduced at rest and increased during stressful situations. Autonomic modulation has been investigated by methods that quantify HR and MAP variability in terms of time and frequency (9). Recently, methods based on non-linear dynamics have been used with interesting results (10). In the present study, we evaluated the time course of MAP and HR and indirectly of autonomic function (by evaluation of MAP and HR variability) in STZ-induced diabetic rats treated with insulin.

Experiments were performed on male Wistar rats from the Animal House of the Universidade Federal do Rio Grande do Sul, Porto Alegre, Brazil, weighing $164.5 \pm 1.7 \mathrm{~g}$ and housed in small groups with free access to tap water and standard rat food. Animals were made diabetic (D) by the injection of STZ (65 $\mathrm{mg} / \mathrm{kg}$, iv) (Sigma Chemical Co., St. Louis, $\mathrm{MO})$ dissolved in citrate buffer, $\mathrm{pH}$ 4.5. Controls (C) were injected with citrate buffer alone. Rats were fasted overnight before STZ injection. STZ-treated rats developed elevated plasma glucose values (confirmed by qualitative measurements of plasma glucose $>300$ $\mathrm{mg} \% 48 \mathrm{~h}$ after the injection) and maintained high urinary glucose levels during the course of the studies. They received daily doses of neutral protamine Hagedorn (NPH) insulin (1 IU, subcutaneously) beginning $48 \mathrm{~h}$ after STZ injection. Metabolic control was evaluated by 24-h urinary glucose measurements and by weighing the animals monthly. Rats were grouped according to the time of evaluation after STZ injection, with 8, 14, 9 and 9 rats being evaluated at 7, 14, 30 and 120 days, respectively.

Catheters filled with saline were implanted under ether anesthesia into the femoral artery and vein (PE-10) for direct measurement of arterial pressure (AP) and drug administration, respectively. One day after catheter placement, the arterial cannula was connected to a strain-gauge transducer (P23Db, Gould-Statham, Oxnard, CA) and blood pressure signals were recorded during a 40-min period with a microcomputer equipped with an analog-to-digital converter board (CODAS, 2-kHz sampling frequency, Dataq Instruments Inc., Akron, OH). Rats were conscious and moved freely during the experiments. Recorded data were analyzed on a beat-to-beat basis. To evaluate MAP variability, we used the standard deviation of the mean of MAP and also a modification of the method proposed by Moraes et al. (10). Three-dimensional return maps (3DRM) were built using as axis $\mathrm{MAP}_{\mathrm{n}} \mathrm{x}$ $\left[\left(\mathrm{MAP}_{\mathrm{n}+1}\right)-\left(\mathrm{MAP}_{\mathrm{n}}\right)\right] \mathrm{x}$ density. The distribution generated was evaluated in order to quantify different patterns of behavior. A set of normalized indices, $\mathrm{P}_{1}, \mathrm{P}_{2}$, and $\mathrm{P}_{3}$, was calculated, with $\mathrm{P}_{1}$ representing the maxi- 
mum concentration of points, $\mathrm{P}_{2}$ the longitudinal axis, and $\mathrm{P}_{3}$ the transversal axis. $\mathrm{P}_{1}$ is inversely proportional to the spread around the maximum concentration cross section, i.e., the mean slope at the most probable MAP. $\mathrm{P}_{2}$ is calculated from the maximum longitudinal length of distribution and represents the dynamic range of MAP variation. The maximum transversal range of variation represented by $\mathrm{P}_{3}$ is the highest beat-to-beat MAP variation. The global behavior was measured by the $M N$ index $\left(M N=P_{1} \times P_{2} \mathrm{x}\right.$ $\left.\mathrm{P}_{3} \times 10^{-3}\right)$. These indices are reported as arbitrary units (AU) (10). HR variability was evaluated in the time domain by the following indices: 1) root mean square of successive difference of RR intervals (RMSSD) and 2) standard deviation of the normal RR intervals (SDNN) (11).

Data are reported as mean \pm SEM, and the Mann-Whitney U-test was used to compare values among groups. The relationship between metabolic control, measured by $24-$ $\mathrm{h}$ urinary glucose and the degree of autonomic modulation, measured by the indices cited above, as well as the relationship between standard deviation of MAP and the $\mathrm{P}_{2}$ index were evaluated by the Spearman rank correlation. Differences were considered to be significant at $\mathrm{P}<0.05$ for all tests.
Urinary glucose levels measured after STZ injection were higher than after citrate buffer injection: $7181 \pm 600 \mathrm{mg} \%$ at 7 days, $8507 \pm 1732 \mathrm{mg} \%$ at 14 days, $5363 \pm 1812$ $\mathrm{mg} \%$ at 30 days and $6697 \pm 2573 \mathrm{mg} \%$ at 120 days in diabetic rats, and $25 \pm 6 \mathrm{mg} \%, 14$ $\pm 4 \mathrm{mg} \%, 13 \pm 6 \mathrm{mg} \%$ and $7 \pm 6 \mathrm{mg} \%$ in controls, at the same times, respectively. The weight of diabetic rats was always lower than that of the control group: $170.5 \pm 7.4 \mathrm{~g}$ at 7 days, $176.8 \pm 29.1 \mathrm{~g}$ at 14 days, $190.6 \pm$ $13.5 \mathrm{~g}$ at 30 days and $318.0 \pm 13.2 \mathrm{~g}$ at 120 days in diabetic rats, and $194.5 \pm 19.1 \mathrm{~g}$, $215.5 \pm 12.4 \mathrm{~g}, 252.4 \pm 14.2 \mathrm{~g}$ and $337.5 \pm$ $30.9 \mathrm{~g}$ in control rats, at the same times, respectively.

Significant cardiovascular changes were found only in 7-day STZ rats, indicating that time-course changes were probably prevented by insulin administration since autonomic dysfunction demonstrated previously after 5-day STZ treatment (8) is also present after 15 and 30 days of untreated STZ diabetes (12). Although MAP did not differ between control and STZ-diabetic rats throughout the experiment, MAP variability evaluated by 3DRM indices was reduced 7 days after STZ administration. $\mathrm{P}_{1}, \mathrm{P}_{2}$, and the global index $\mathrm{MN}$ were significantly reduced in diabetic rats (Table 1). Two examples of

Table 1 - Cardiovascular characterization of diabetic (D) and control (C) rats 7, 14, 30 and 120 days after streptozotocin injection.

Data are reported as means \pm SEM. ${ }^{*} \mathrm{P} \leq 0.05$ vs control rats (Mann-Whitney U-test). HR $=$ Heart rate; RMSSD $=$ root mean square of successive difference of RR intervals; $S D N N=$ standard deviation of the normal RR intervals; $M A P=$ mean arterial pressure; $S D=$ standard deviation; $A U=$ arbitrary units.

\begin{tabular}{|c|c|c|c|c|c|c|c|c|}
\hline & \multicolumn{2}{|c|}{7 days } & \multicolumn{2}{|c|}{14 days } & \multicolumn{2}{|c|}{30 days } & \multicolumn{2}{|c|}{120 days } \\
\hline & C & $\mathrm{D}$ & C & $\mathrm{D}$ & C & D & C & D \\
\hline HR (bpm) & $381 \pm 13$ & $319 \pm 20^{*}$ & $388 \pm 8$ & $379 \pm 26$ & $380 \pm 22$ & $348 \pm 16$ & $365 \pm 20$ & $342 \pm 20$ \\
\hline RMSSD (ms) & $5 \pm 1$ & $12 \pm 2$ & $4 \pm 1$ & $4 \pm 1$ & $7 \pm 2$ & $5 \pm 1$ & $6 \pm 1$ & $5 \pm 1$ \\
\hline SDNN (ms) & $12 \pm 2$ & $20 \pm 3^{*}$ & $14 \pm 3$ & $12 \pm 3$ & $13 \pm 1$ & $15 \pm 1$ & $15 \pm 2$ & $14 \pm 3$ \\
\hline $\mathrm{MAP}(\mathrm{mmHg})$ & $118 \pm 8$ & $120 \pm 7$ & $123 \pm 4$ & $108 \pm 12$ & $115 \pm 7$ & $104 \pm 14$ & $127 \pm 6$ & $101 \pm 14$ \\
\hline SD of MAP & $5 \pm 0.03$ & $8 \pm 0.1$ & $7 \pm 0.1$ & $7 \pm 0.02$ & $6 \pm 0.2$ & $7 \pm 0.1$ & $9 \pm 0.2$ & $10 \pm 0.1$ \\
\hline$P_{1}(A U)$ & $61 \pm 5$ & $52 \pm 2^{*}$ & $67 \pm 2$ & $56 \pm 6$ & $60 \pm 4$ & $60 \pm 2$ & $59 \pm 6$ & $60 \pm 2$ \\
\hline$P_{2}(A U)$ & $41 \pm 0.3$ & $29 \pm 2^{*}$ & $37 \pm 1$ & $37 \pm 5$ & $34 \pm 2$ & $31 \pm 5$ & $44 \pm 10$ & $35 \pm 3$ \\
\hline$P_{3}(A U)$ & $67 \pm 6$ & $51 \pm 4$ & $64 \pm 2$ & $50 \pm 3$ & $65 \pm 3$ & $65 \pm 11$ & $72 \pm 23$ & $57 \pm 7$ \\
\hline $\mathrm{MN}(\mathrm{AU})$ & $171 \pm 30$ & $77 \pm 10^{*}$ & $157 \pm 12$ & $107 \pm 21$ & $139 \pm 14$ & $132 \pm 47$ & $200 \pm 82$ & $124 \pm 29$ \\
\hline
\end{tabular}


Figure 1 - Left panel, Scattergram representing 24-h urinary glucose and the MN index of diabetic and control rats 7 days after streptozotocin. Right panel, A three-dimensional return map of one control and one diabetic rat.

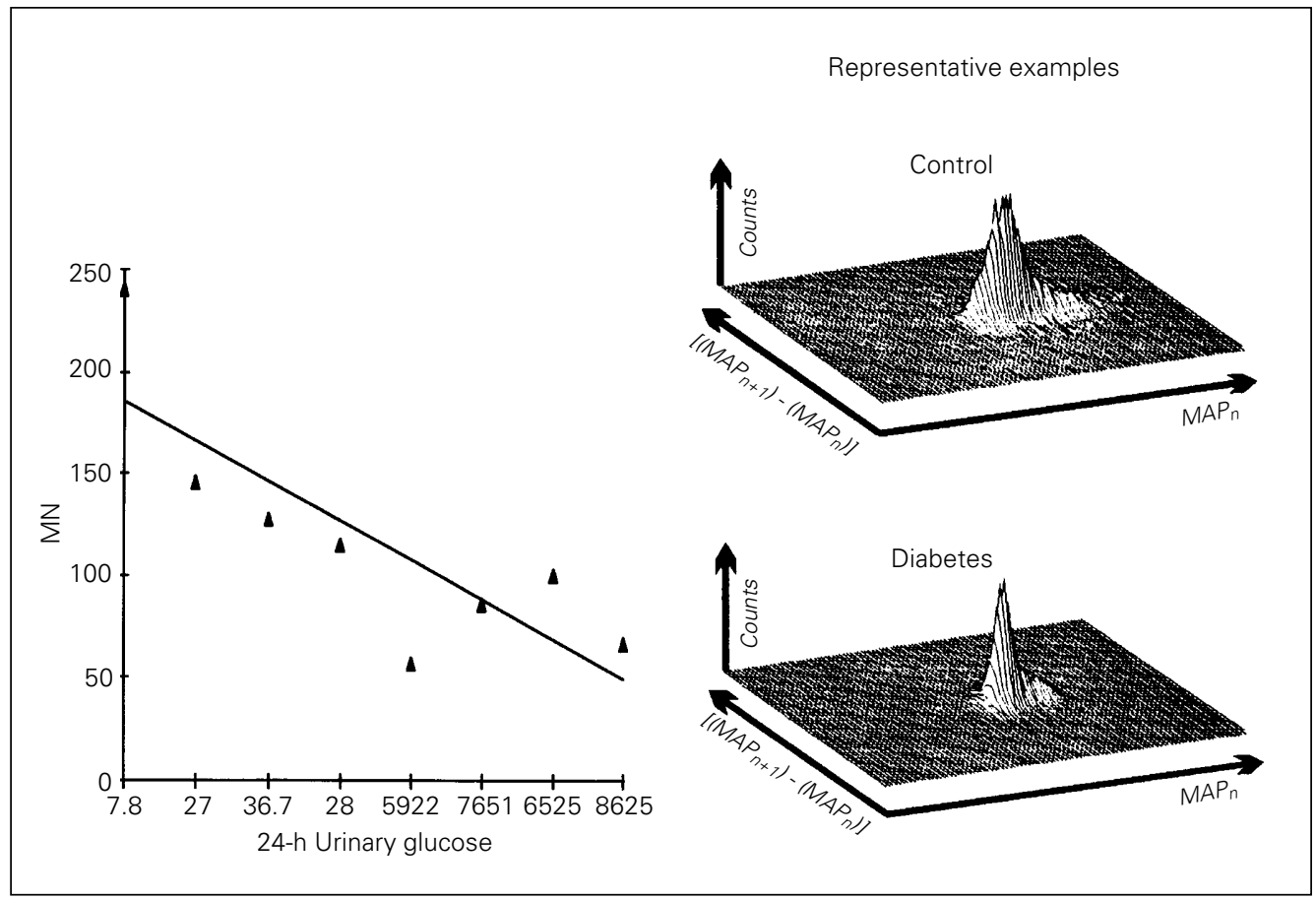

3DRM, one for a diabetic and the other for a control rat, are shown in Figure 1. The mean of the standard deviations of MAP was $8 \pm$ 0.1 in diabetics and $5 \pm 0.03$ in controls $(\mathrm{P}<0.05)$ (Table 1). There was a positive correlation between the standard deviation of MAP and the $\mathrm{P}_{2}$ index obtained by the 3DRM $(r=0.94, \mathrm{P}<0.002)$.

Seven-day diabetes significantly reduced resting HR from $381 \pm 13$ to $319 \pm 20 \mathrm{bpm}$ while these differences were not found 14 , 30 and 120 days after induction of diabetes in rats treated with insulin (Table 1). HR variability, reported as SDNN, was increased in diabetic rats $(20 \pm 3 \mathrm{~ms})$ compared to controls (12 $\pm 2 \mathrm{~ms}$ ), although RMSSD did not change. There was a negative correlation between 24-h urinary glucose and resting $\mathrm{HR}(\mathrm{r}=-0.76, \mathrm{P}=0.03)$, as well as between the former and $\mathrm{MN}$ index $(\mathrm{r}=-0.83, \mathrm{P}=$ 0.01) (Figure 1). All indices described above were similar for STZ-treated rats at 14, 30 and 120 days, and controls (Table 1).

Several studies have demonstrated changes in AP as well as increased or decreased baroreflex responses to changes in blood pressure in experimental diabetes (1315). We have previously demonstrated that 5-day STZ diabetes in rats, with no insulin administration, not only reduces AP and HR, but also reduces intrinsic $\mathrm{HR}$, vagal function and sympathetic tone (8). In the present study we found that 7-day STZ-diabetic rats presented reduced MAP variability, reduced HR and increased HR variability. These different findings may indicate that insulin administration in this early phase of diabetes contributes to this improved state. Indeed, the normalization of spontaneous beating rate accounted for by the improvement of glucose oxidation in STZ rats observed by other investigators (16) indicates the role of insulin replacement in changing the natural history of cardiovascular dysfunction in this experimental model. Moreover, other experiments from our laboratory have demonstrated that hypotension and bradycardia, that may represent disautonomy, are present in this model at 5 and 14 days after STZ injection if the animals are not treated with insulin $(12,17)$. Decreased resting HR, increased HR variability, as well as decreased 
MAP variability, may be related to changes in autonomic function at this time. Lower resting $\mathrm{HR}$ has been attributed to a change in sinoatrial node as well as to functional changes in cardiac cholinergic mechanisms (8). Chang and Lund (13) also observed that insulin treatment restores normal cardiovascular and baroreflex function, at least when given during the early stages of diabetes (12week experiment). In contrast, baroreflex sensitivity changes from hypersensitivity to a later state of hyposensitivity indicating that the reduced ability to regulate HR is attributable to an impairment of cardiac parasympathetic regulation $(8,13)$. In a morphological study of the autonomic nervous system, Schmidt et al. (18) showed amelioration of neuroaxonal dystrophy by early treatment of diabetic rats with insulin, by pancreatic islet transplant or by administration of the aldosereductase inhibitor sorbinyl, but not by dietary administration of myo-inositol. However, when these treatments were instituted later, after neuropathy was established, inhibition of progression was attained, but the parameters studied did not normalize (19).

These findings are in agreement with our results since the changes in resting HR persisted while MAP and HR variability improved at 7 days and returned to baseline values after this time, indicating that timedependent changes of metabolic parameters due to hyperglycemia and insulinopenia, or decreases in cardiac adrenergic receptors (20) may be possible explanations for the opposite findings. The controversial data suggesting changes induced by diabetes may be attributed to differences in experimental and analytical approaches. The time after diabetes induction, age of the animal, diabetogen used and the use of insulin could modify the diabetic state obtained, changing the natural history of different complications of experimental diabetes (14).

Since the neurotoxic effects of STZ could be expected during the first weeks after its injection, with regenerative changes after 6 weeks (4), we cannot attribute any toxic effect of the drug to our results, because they indicate amelioration of autonomic function at 7 days of diabetes.

Indeed, changes in autonomic function at 7-day STZ diabetes did not persist, at least until 4 months of diabetes, since the evaluation at 14, 30 and 120 days after STZ injection was similar for diabetic and control animals.

In 7-day STZ rats, but not in other groups, we found a strong negative correlation between urinary glucose and the resting HR and MN, a global index of MAP variability. Although urinary glucose levels did not fall in diabetics with the progression of time (even when they received daily insulin administration), higher insulin levels are expected to occur on the first days after STZ injections (21). However, it seems that autonomic changes may be correlated with the metabolic control or diabetes per se and are not related to the direct action of STZ. Results obtained by Schmidt et al. (22), who studied rats treated with STZ receiving insulin, and by Chang and Lund (13), who studied rats which received STZ and did not develop diabetes, strongly suggest that the lesions demonstrated by these investigators in STZ-induced diabetic animals were produced by diabetes and were not the result of a direct toxic effect of the diabetogenic agent.

We conclude that rats with short-term diabetes mellitus induced by STZ 7 days before, and treated with insulin, presentd an improvement in autonomic control of HR and MAP. The metabolic control may influence these results since during the first 7 days after induction of diabetes glucose was correlated with parameters of autonomic function. This result suggests that insulin treatment and the better metabolic control in this model prevented or abolished the timedependent changes in arterial pressure, HR and MAP variability. 


\section{References}

1. Sampson MJ, Wilson $S$, Karaggianis $P$, Edmonds ME \& Watkins PJ (1990). Progression of diabetic autonomic neuropathy over a decade in insulin dependent diabetics. Quarterly Journal of Medicine, 75: 635-646.

2. Murray A, Ewing DJ, Campbell IW, Neilson JMM \& Clarke BF (1975). RR interval variations in young male diabetics. British Heart Journal, 37: 882-885.

3. Rubler S, Chu DA \& Bruzzone CL (1985). Blood pressure and heart rate responses during 24-hour ambulatory monitoring and exercise in men with diabetes mellitus. American Journal of Cardiology, 55: 801806.

4. Monckton G \& Pehowich E (1980). Autonomic neuropathy in the streptozotocin diabetic rat. Journal Canadien des Sciences Neurologiques, 7: 135-142.

5. Schmidt RE \& Plurad SB (1986). Ultrastructural and biochemical characterization of autonomic neuropathy in rats with chronic streptozotocin diabetes. Journal of Neuropathology and Experimental Neurology, 45: 525-544.

6. Yagihashi S \& Sima AAS (1985). Diabetic autonomic neuropathy in the BB rat: UItrastructural and morphometric changes in sympathetic nerves. Diabetes, 34: 558564.

7. Yagihashi S \& Sima AAS (1986). Diabetic autonomic neuropathy in the BB rat: UItrastructural and morphometric changes in parasympathetic nerves. Diabetes, 35: 733-743.

8. Maeda CY, Fernandes TG, Timm HB \& Irigoyen MC (1995). Autonomic dysfunction in short-term experimental diabetes. Hypertension, 26 (Part 2): 1000-1004.

9. Parati G, Saul JP, Di Rienzo M \& Mancia G (1995). Spectral analysis of blood pressure and heart rate variability in evaluating cardiovascular regulation: A critical appraisal. Hypertension, 25: 1276-1286.
10. Moraes R, Ferlin E, Polanczik C, Wainstein M, Zaslawski L \& Ribeiro J (1993). Three dimension return map: a method for quantification of heart rate variability. Journal of the American College of Cardiology, 21 (Suppl 2): 156 (Abstract).

11. Hayano J, Sakakibara $Y$, Yamada A, Yamada M, Mukai S, Fujinami T, Yokoyama K, Watanabe $Y$ \& Takata $K$ (1991). Accuracy of assessment of cardiac vagal tone by heart rate variability in normal subjects. American Journal of Cardiology, 67: 199-204.

12. Dall'Ago P, Fernandes TG, Machado UF, Belló AA \& Irigoyen MC (1997). Baroreflex and chemoreflex dysfunction in streptozotocin-diabetic rats. Brazilian Journal of Medical and Biological Research, 30: 119124.

13. Chang KSK \& Lund DD (1986). Alterations in the baroreceptor reflex control of heart rate in streptozotocin diabetic rats. Journal of Molecular and Cellular Cardiology, 18: 617-624.

14. Jackson CV \& Carrier GO (1983). Influence of short-term experimental diabetes on blood pressure and heart rate in response to norepinephrine and angiotensin II in the conscious rat. Journal of Cardiovascular Pharmacology, 5: 260-265.

15. Homma S, Yamazaki $Y$ \& Karakida $T$ (1993). Blood pressure and heart rate relationships during cervical sympathetic and vagus nerve stimulation in streptozotocin diabetic rats. Brain Research, 629: 342-344.

16. Nicholl TA, Lopaschuk GD \& MacNeill JH (1991). Effects of free fatty acids and dichloroacetate on isolated working diabetic rat heart. American Journal of Physiology, 261: H1053-H1059.
17. Maeda CY, Fernandes TG, Lulhier F \& Irigoyen MC (1995). Streptozotocin diabetes modifies arterial pressure and baroreflex sensitivity in rats. Brazilian Journal of Medical and Biological Research, 28: 497-501.

18. Schmidt RE, Plurad SB, Sherman WR, Williamson JR \& Tilton RG (1989). Effects of aldose-reductase on neuroaxonal dystrophy and levels of myo-inositol and sorbitol in sympathetic autonomic ganglia of streptozotocin-induced diabetic rats. Diabetes, 38: 569-579.

19. Schmidt RE, Plurad SB, Coleman BD, Williamson JR \& Tilton RG (1991). Effects of sorbinyl, dietary myo-inositol supplementation, and insulin on resolution of neuroaxonal dystrophy in mesenteric nerves of streptozotocin-induced diabetic rats. Diabetes, 40: 574-582.

20. Williams RS, Schaible TF, Scheur J \& Kennedy R (1983). Effects of experimental diabetes on adrenergic and cholinergic receptors of rat myocardium. Diabetes, 32: 881-886.

21. Junod A, Lambert $A E$, Stauffacher $W$ \& Renold AE (1969). Diabetogenic action of streptozotocin: relationship of dose to metabolic response. Journal of Clinical Investigation, 48: 2129-2139.

22. Schmidt RE, Plurad SB, Olack B \& Scharp DW (1983). The effect of pancreatic islet transplantation and insulin therapy on experimental diabetic autonomic neuropathy. Diabetes, 32: 532-540. 